\title{
Author Correction: Visualizing flow in an intact CSF network using optical coherence tomography: implications for human congenital hydrocephalus
}

\section{Priya Date, Pascal Ackermann, Charuta Furey, Ina Berenice Fink, Stephan Jonas $\mathbb{D}$, Mustafa K. Khokha, KristopherT. Kahle \& Engin Deniz}

Correction to: Scientific Reports https://doi.org/10.1038/s41598-019-42549-4, published online 17 April 2019

In this Article, the Supplementary Information movies are incorrectly referenced. As a result, in the Results section under the subheading 'Loss of cilia motility or biogenesis causes discrete ventricular phenotypes',

“These tadpoles demonstrated no or very slow CSF flow velocities as detected by OCT imaging (Fig. 3b,c).”

should read:

“These tadpoles demonstrated no or very slow CSF flow velocities as detected by OCT imaging (Fig. 3b,c - Movie 4)."

Additionally, in the same section,

"We observed a dramatic loss of flow throughout the ventricles, but unlike the 221 orf59 knockdown, the ventricles were significantly larger, resembling communicating hydrocephalus (Figs. 3d,e, S2b, S3a)."

should read:

"We observed a dramatic loss of flow throughout the ventricles, but unlike the 221 orf59 knockdown, the ventricles were significantly larger, resembling communicating hydrocephalus (Figs. 3d,e, S2b,S3a - Movie 5)."

In the Results section under the subheading 'Loss of L1CAM function in Xenopus tadpoles causes aqueductal stenosis',

"Next, we investigated the ventricular CSF flow and mapped FF1 to FF5, which appeared intact (Figs. 4e,f, S2c, Movie 5)."

should read:

"Next, we investigated the ventricular CSF flow and mapped FF1 to FF5, which appeared intact (Figs. 4e,f, S2c, Movie 6)."

In the Results section under the subheading 'CRB2 loss-of-function leads to cerebral aqueduct stenosis and ventriculomegaly in humans and frogs',

"Importantly, the flow of the lateral - $3^{\text {rd }}$ (FF1 and 2) and midbrain ventricles (FF3) was severely affected (Figs. 5f,g, S2d, Movie 6), consistent with a role in cilia. Interestingly, we did not see any changes in the flow of the fourth ventricle (FF4 and FF5) (Fig. 5f,g, Movie 6), whether the flow in these posterior flow fields reflects near normal cilia function or choroid plexus flow remains to be determined." 
should read:

"Importantly, the flow of the lateral - $3^{\text {rd }}$ (FF1 and 2) and midbrain ventricles (FF3) was severely affected (Figs. 5f,g, S2d, Movie 7), consistent with a role in cilia. Interestingly, we did not see any changes in the flow of the fourth ventricle (FF4 and FF5) (Fig. 5f,g, Movie 7), whether the flow in these posterior flow fields reflects near normal cilia function or choroid plexus flow remains to be determined."

(c) (i) Open Access This article is licensed under a Creative Commons Attribution 4.0 International License, which permits use, sharing, adaptation, distribution and reproduction in any medium or format, as long as you give appropriate credit to the original author(s) and the source, provide a link to the Creative Commons license, and indicate if changes were made. The images or other third party material in this article are included in the article's Creative Commons license, unless indicated otherwise in a credit line to the material. If material is not included in the article's Creative Commons license and your intended use is not permitted by statutory regulation or exceeds the permitted use, you will need to obtain permission directly from the copyright holder. To view a copy of this license, visit http://creativecommons.org/licenses/by/4.0/.

(c) The Author(s) 2020 